\title{
High $\mathbf{p}_{\mathrm{T}}$ Probes in PbPb Collisions at ATLAS
}

\section{Ewelina Maria LOBODZINSKA* on behalf of the ATLAS Collaboration}

E-mail: ewelina.maria.lobodzinska@cern.ch

Using the ATLAS detector several studies of the jet quenching effect in the hot, dense medium produced in the lead-lead collisions, at the center of mass energy $\sqrt{\mathrm{s}_{\mathrm{NN}}}=2.76 \mathrm{TeV}$, at LHC were made. Different observables were investigated. The jet yield suppression with the increasing centrality was observed both in the dijet and the inclusive jets analyses. Path length dependence of the jet energy loss was also studied and shown to have a big effect on the jet suppression. Electroweak bosons $(Z, \gamma)$ were shown to be unaffected by the quark-gluon plasma, so could be used as an energy calibration scale for associated production with jets. The energy imbalance in the $\gamma$-jet and $Z$-jet pairs was observed to strongly increase with centrality.

The European Physical Society Conference on High Energy Physics -EPS-HEP2013

18-24 July 2013

Stockholm, Sweden

${ }^{*}$ Speaker. 


\section{Introduction and first evidence of jet quenching in ATLAS}

Collisions of heavy ions at ultrarelativistic energies are expected to produce a hot, dense, strongly interacting matter, in which quarks and gluons become locally deconfined. In this medium high-energy quarks and gluons are expected to transfer energy to the medium by multiple interactions with the ambient plasma. There is a reach theoretical literature describing experimental effects expected in connection with the quark-gluon plasma. Already Bjorken proposed to look for "jet quenching" in proton-proton collisions [1]. He also proposed the analysis in which the quenching could be looked for i.e. in the analysis of dijets where one of the jets is produced at the periphery of the collisions. These highly unbalanced in energy jets would be a probe confirming the creation of quark-gluon plasma. Experiments to probe quark-gluon plasma were performed at RHIC (Relativistic Heavy Ion Collider) e.g. [2]. However, the jet results from RHIC were not really conclusive due to limited rapidity coverage of the experiment and jet energies comparable to the underlying event energy. The LHC heavy ion (HI) program was prepared to provide an opportunity to study jet quenching at much higher energies. Already at the end of 2010 the ATLAS Collaboration published first results on the measurement of "jet quenching" in dijets analysis [4]. The data used for the study were collected in lead-lead $(\mathrm{Pb}-\mathrm{Pb})$ collisions at $\sqrt{s_{N N}}=2.76 \mathrm{TeV}$ per nucleon-nucleon pair and correspond to an integrated luminosity of $1.7 \mu \mathrm{b}^{-1}$. Analysed were dijets of highest transverse energy in events where those jets have an azimuthal angle separation of $\Delta \varphi>\pi / 2$. The asymmetry between jets was defined by the following formula:

$$
A_{J}=\frac{E_{T 1}-E_{T 2}}{E_{T 1}+E_{T 2}}, \quad \Delta \varphi>\frac{\pi}{2}
$$

The leading jet must have the transverse energy $E_{T 1}>100 \mathrm{GeV}$ and the subleading one $E_{T 2}>25$ $\mathrm{GeV}$. The parameter useful in heavy ion collisions is centrality, which tells if the collision was more central or rather peripheral. Centrality is related to the overlap volume of the two colliding nuclei and can be expressed by the impact parameter, the number of participant nucleons and the average number of binary collisions. In ATLAS [3] the centrality is measured with the forward calorimeter: the higher transverse energy collected, the more central the event. [4]
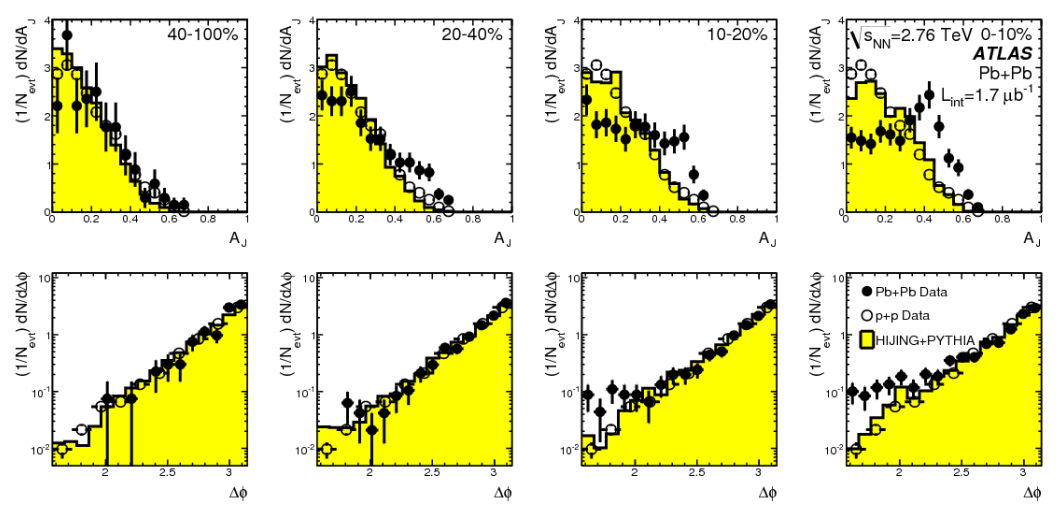

Figure 1: Top: Asymmetry distribution for different centrality bins. Bottom: $\Delta \varphi$ distribution for different centrality bins. $\mathrm{Pb}-\mathrm{Pb}$ data are marked with black points, $p-p$ data with empty points and the histograms show Hijing MC with overlaid dijet events from PYTHIA [4]. 
The distribution of the dijet asymmetry is shown in Fig. 1 for four centrality bins. It can be noticed that for the peripheral bins the asymmetry for $\mathrm{Pb}-\mathrm{Pb}$ data is in a very good agreement with the Monte Carlo (MC) predictions (unquenched Hijing with overlayed dijet PYTHIA events) and the measurement for $p$ - $p$ data. However, with increasing centrality the asymmetry distribution for $\mathrm{Pb}-\mathrm{Pb}$ data broadens, the mean shifts to higher values and the peak at zero disappears. None of these is observed for $p$ - $p$ data or MC predictions. In the same figure also the distributions of $\Delta \varphi$ are shown. They show that leading and subleading jets are mainly back-to-back, however with increasing centrality a systematic increase is observed in the rate of the subleading jets at large angles relative to the recoil direction.

This observation of changes in asymmetry distributions with increasing centrality is explained as a jet quenching in a hot, dense medium.

\section{Inclusive jet suppression}

The - already historical - observation of highly asymmetric dijets in central $\mathrm{Pb}-\mathrm{Pb}$ collisions discussed in the previous section can be understood, as "differential" jet quenching, where one parton produced in the original hard scattering loses significantly more energy than the other. The reason for this may be a different path length of the partons in the medium. In such a measurement we are not sensitive to quenching effects when both jets lose a comparable amount of energy, so an inclusive study is needed. It was performed [5] using heavy ion data from 2010 with an integrated luminosity of $7 \mu \mathrm{b}^{-1}$. Measured was the central to peripheral ratio defined as:

$$
R_{C P}^{\text {meas }}\left(p_{T}\right)=\frac{1}{R_{\text {coll }}^{\text {cent }}} \frac{\frac{N_{j \text { jet }}^{\text {cent }}\left(p_{T}\right)}{N_{\text {evt }}^{\text {cent }}}}{N_{\text {jet }}^{60-80}\left(p_{T}\right)}
$$

where $N_{\text {jet }}^{\text {cent }}\left(p_{T}\right)$ represents the measured jet yield in a given $p_{T}$ and centrality bin, and $N_{\text {evt }}^{c e n t}$ and $N_{\text {evt }}^{60-80}$ are the number of $\mathrm{Pb}-\mathrm{Pb}$ collisions in the chosen and peripheral reference centrality intervals, respectively. $R_{\text {coll }}^{\text {cent }}=\left\langle N_{\text {coll }}^{\text {cent }}\right\rangle /\left\langle N_{\text {coll }}^{60-80}\right\rangle$ where $\left\langle N_{\text {coll }}^{\text {cent }}\right\rangle$ and $\left\langle N_{\text {coll }}^{60-80}\right\rangle$ are the overall average number of collisions and average number of collisions in the $60-80 \%$ centrality bin, respectively.

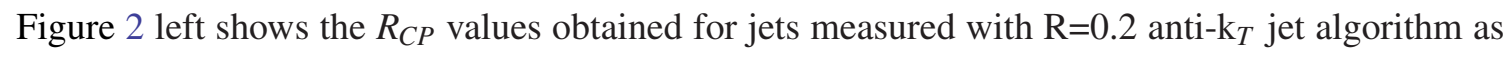
a function of $p_{T}$ in several bins of centrality. The variation with transverse momentum is observed to be at most weak. For the most central bin we observe a factor of two suppression in jets yields compared to the most peripheral bin (60-80\%), while for the bin with centrality 50-60\% the ratio is around 0.9.

The right plot of Fig. 2 shows the $R_{C P}$ as a function of jet radius for $89<p_{T}<103 \mathrm{GeV}$ and four centrality bins. A weak - but still significant - variation of jet yields with jet radius is observed. $R_{C P}$ seems to increase gradually from $\mathrm{R}=0.2$ to $\mathrm{R}=0.5$. It is expected that the jet quenching effect should strongly depend on the path length of the parton shower in the medium. Since the quarkgluon plasma created in the non-central $\mathrm{Pb}-\mathrm{Pb}$ collisions has an approximately elliptic profile in the transverse plane, the measurement of the dependence on the path length can be realised by studying the $R_{C P}$ dependence on the azimuthal angle- $\Delta \phi$ - with respect to the elliptic flow event plane - see Fig. 3. 

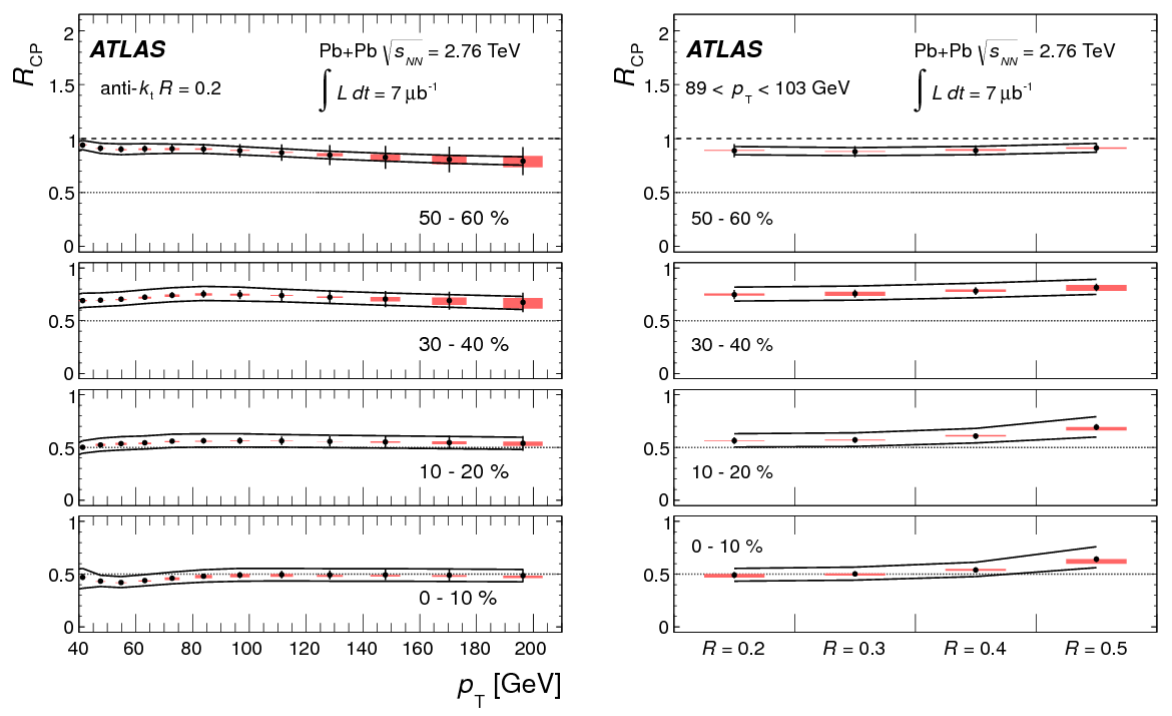

Figure 2: Left: $R_{C P}$ as a function of $p_{T}$ for jet algorithm radius $\mathrm{R}=0.2$ in four centrality bins. Right: $R_{C P}$ as a function of jet radius for fixed $p_{T}$ interval and four centrality bins. For all plots: error bars indicate statistical errors, the shaded boxes indicate point-to-point systematic errors that are only partially correlated. The solid lines indicate systematic errors that are fully correlated between points [5].

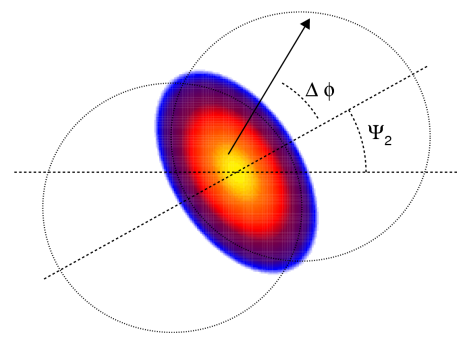

Figure 3: Illustration of the elliptic profile of the overlap region in non-central $\mathrm{Pb}-\mathrm{Pb}$ collisions. $\Psi_{2}$ describes the orientation of the minor axis of the ellipse with respect to the horizontal plane. The arrow shows the parton path in the transverse plane of the hypothetical jet. $\Delta \phi$ is the angle of the jet relative to $\Psi_{2}$.

To investigate the azimuthal dependence of inclusive jet suppression, ATLAS has measured two observables: 1.) $v_{2}^{\text {jet }}$ - which characterises the fractional variation of jet yield with $\Delta \phi$ at a given $p_{T}$ and 2.) the ratios of inclusive jet spectra measured over different $\Delta \phi$ ranges [6]. Figure 4 shows $v_{2}^{\text {jet }}$ as a function of $p_{T}$ for six centrality bins. Statistically significant non-zero values are observed for $p_{T}<160 \mathrm{GeV}$, for all centrality bins.

To measure the second observable, the ratio $\mathrm{R}_{\Delta \phi}$ was defined as

$$
R_{\Delta \phi}=\frac{\left.\frac{d^{2} N_{j e t}}{d p_{T} d \Delta \phi}\right|_{\Delta \phi=\Delta \phi_{i}}}{\left.\frac{d^{2} N_{j e t}}{d p_{T} d \Delta \phi}\right|_{\Delta \phi=\Delta \phi_{j}}}
$$

where $\Delta \phi_{j}$ is the interval closest to the minor elliptic axis i.e. $0<\Delta \phi<\pi / 8$. The distribution of 

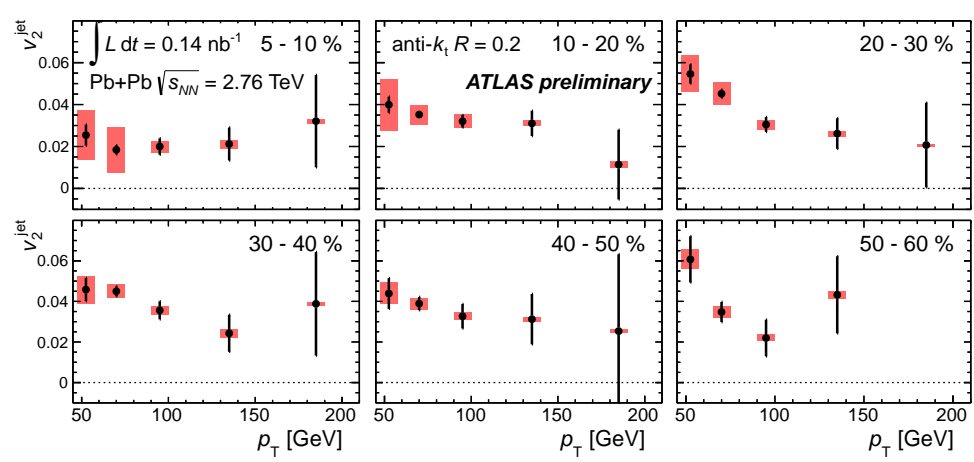

Figure 4: $v_{2}^{\text {jet }}$ as a function of $p_{T}$ for different centrality bins. Error bars show statistical errors and shaded area systematic uncertainties[6].

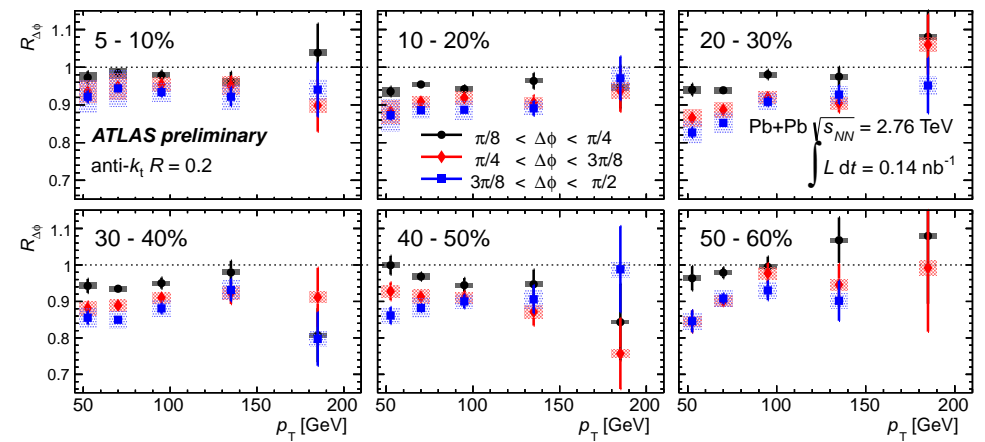

Figure 5: $R_{\Delta \phi}$ as a function of $p_{T}$ for three $\Delta \phi$ intervals for six centrality bins. Error bars show statistical errors, shaded boxes systematic uncertainties [6].

$R_{\Delta \phi}$ as a function of $p_{T}$ is shown in Fig. 5 for six centrality bins. A clear reduction of jet yields with increasing $\Delta \phi$ is observed.

\section{Electroweak bosons in $\mathrm{HI}$ collisions}

The kinematics of $\gamma, Z$ and $W$ - since they are colourless - should not be affected by the quarkgluon plasma and so they can provide a mean for understanding the interactions of colour sensitive objects with the medium. Analysing events with a jet and a penetrating probe, such as photon or $W / Z$ bosons, offers the possibility of calibrating the energy of the initial jet. In the first step, however, photon $/ W / Z$ rates should be measured and the potential interactions with the quark-gluon plasma investigated. ATLAS has measured $\gamma, W$ and $Z$ boson yields [7] [8] [9]. In Fig. 6 centrality dependence (displayed as a dependence on average number of participant nucleons $N_{\text {part }}$ ) of photon yields per event for different $p_{T}$ bins (top left figure) and $Z$ boson yields per event for $e e$ and $\mu \mu$ decay channels (top right figure) are shown.

No centrality dependence of $\gamma$ or $Z$ boson yields is observed. These observations indicate that $\gamma$ and $Z$ bosons are unaffected by the medium in HI collisions. This is surmised to imply that the energy loss mechanism only affects particles with colour charge in the quark-gluon plasma, however the detailed understanding is still unclear. A pair of the colour neutral boson and the 

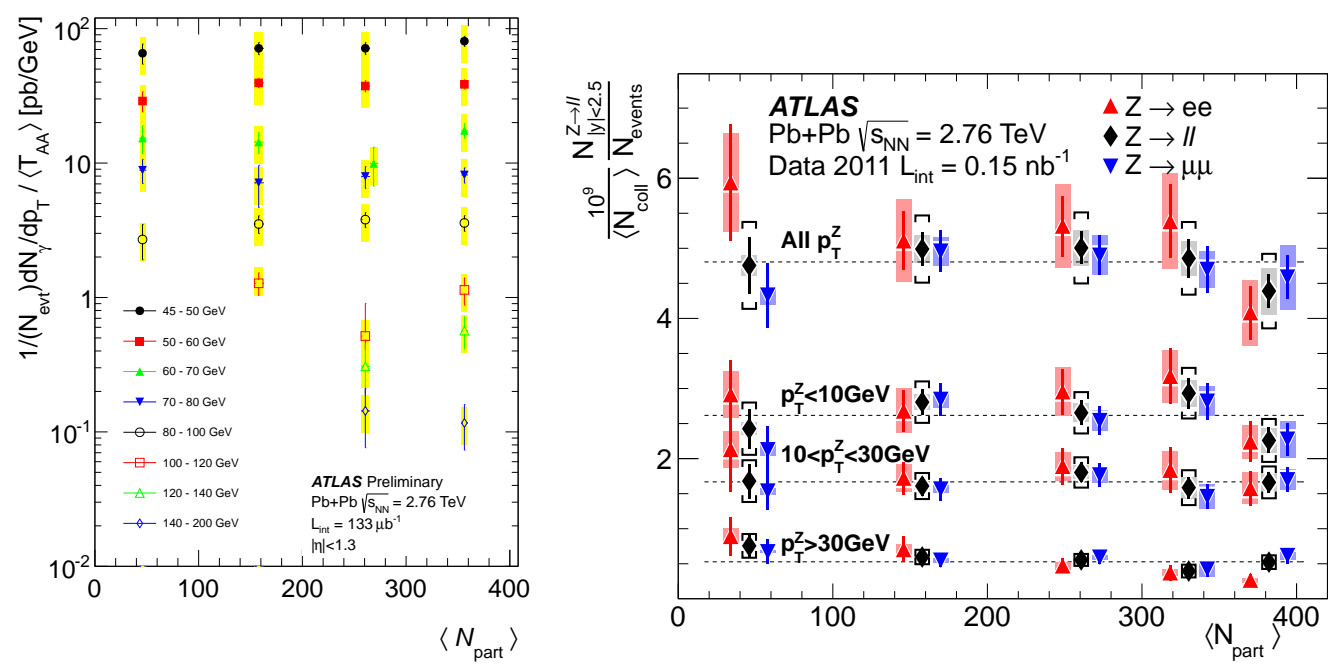

Figure 6: Centrality dependence of ( left) the photon yield per event for different $p_{T}$ bins and of (right) $Z$ boson yields normalised by the total number of collisions: In all plots: bars and boxes represent statistical and systematic uncertainties, respectively [8][9].
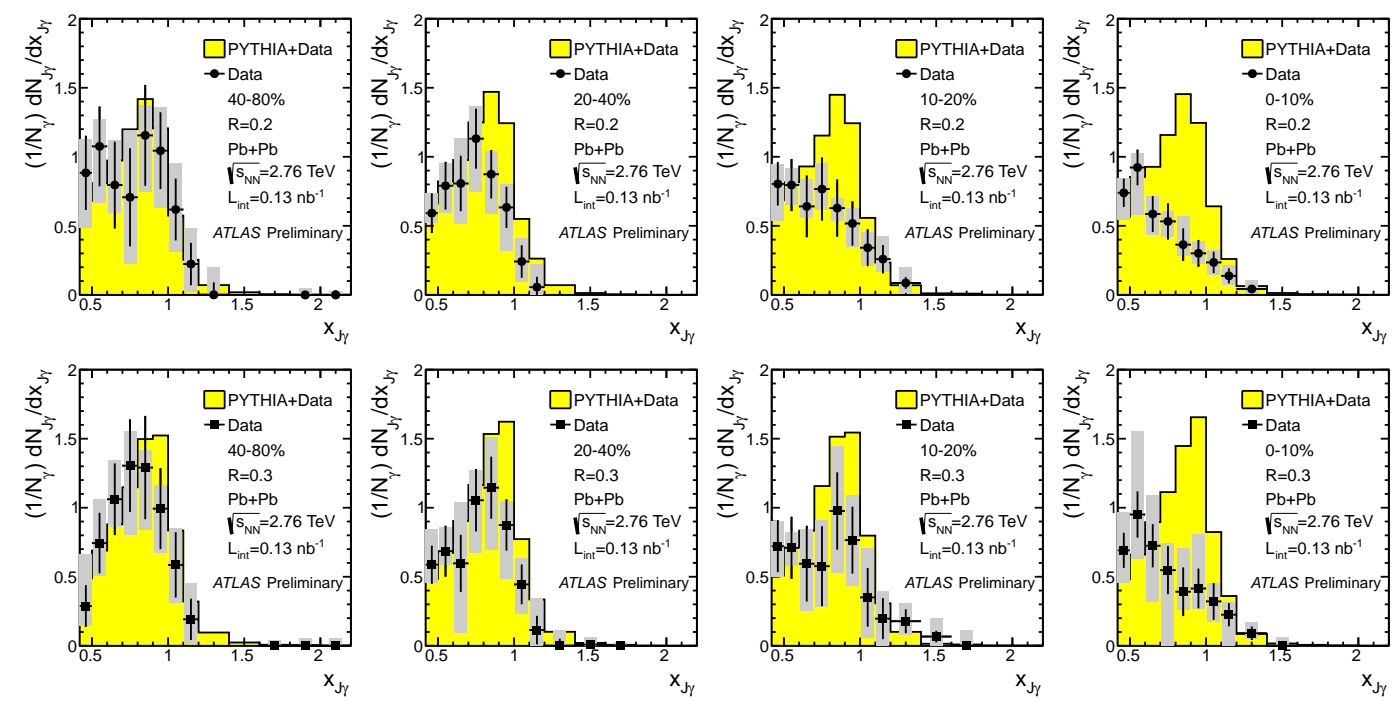

Figure 7: Distributions of $x_{J \gamma}$ for four centrality bins for two jet radii: $\mathrm{R}=0.2$ (top) and $\mathrm{R}=0.3$ (bottom). Statistical errors marked by error bars, systematic by shaded blocks. PYTHIA MC (with data overlay) predictions shown with a histogram [10].

colour sensitive jet composes a probe that has been called a "golden channel" for understanding the interactions of colour sensitive objects in the quark-gluon plasma. ATLAS has investigated jets and high $p_{T}$ prompt photon correlations [10] by analising the ratio of jet transverse energy to photon transverse energy $x_{J \gamma}=p_{T}^{J} / p_{T}^{\gamma}$ for different centrality bins. The distribution of $x_{J \gamma}$ normalised to the number of photons, compared to PYTHIA (with overlaid data), is shown in Fig. 7. For peripheral bins the data agree with the MC predictions. However, as the centrality increases there is a shift in the distribution towards smaller $x_{J \gamma}$ suggesting that more and more jets fall below the $x_{J \gamma}$ cut $\left(x_{J \gamma}>\right.$ 


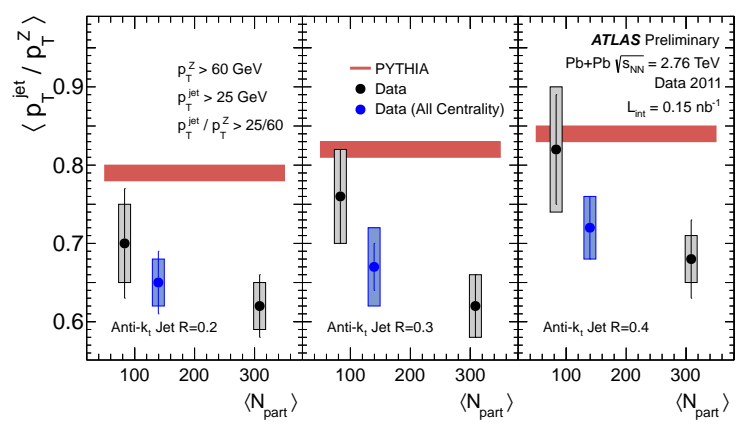

Figure 8: Means from the $p_{T}^{\text {jet }} / p_{T}^{Z}$ distributions as a function of the average number of participants. Error bars represent statistical and boxes systematic uncertainties. The band shows PYTHIA predictions [11].

25/60). A similar analysis was performed for $Z$ boson + jet pairs [11]. Figure 8 shows the means of the jet to $Z$ boson transverse momentum ratios $\left\langle p_{T}^{j e t} / p_{T}^{Z}\right\rangle$ for two centrality bins (peripheral and more central), measured for different jet radii and compared to PYTHIA predictions. The data clearly indicate suppression in the extracted mean ratio $p_{T}^{\text {jet }} / p_{T}^{Z}$ compared to PYTHIA and its increase for higher centrality.

\section{Conclusions}

The jet quenching observed in ATLAS in 2010 was investigated by means of other high $p_{T}$ probes. A factor of two suppression in jet yields in central to peripheral collisions was observed for inclusive jet production. A reduction of jet yields was also seen for increasing path length of a jet in the quark-gluon plasma. In agreement with expectations, electroweak bosons were shown not to interact with the hot, dense medium created in the HI collisions. Energy imbalance in $\gamma$ - jet and $Z$ -jet events tends to increase with centrality. Interesting results are also expected from the analysis of high $p_{T}$ probes in $p-\mathrm{Pb}$ data.

\section{References}

[1] J. D. Bjorken, FERMILAB Report No. FEBMILAB-PUB-82-059-THY, 1982.

[2] PHENIX Collaboration, Phys. Rev. Lett. 88, 022301 (2001). STAR Collaboration, Phys. Rev. Lett. 89, 202301 (2002).

[3] ATLAS Collaboration, JINST 3, S08003 (2008).

[4] ATLAS Collaboration, Phys. Rev. Lett. 105, 252303 (2010).

[5] ATLAS Collaboration, Phys. Lett. B 719, 220 (2013).

[6] ATLAS Collaboration, ATLAS-CONF-2012-16.

[7] ATLAS Collaboration, ATLAS-CONF-2011-078.

[8] ATLAS Collaboration, ATLAS-CONF-2012-051.

[9] ATLAS Collaboration, Phys. Rev. Lett. 110, 022301 (2013).

[10] ATLAS Collaboration, ATLAS-CONF-2012-121.

[11] ATLAS Collaboration, ATLAS-CONF-2012-119. 